\title{
Adolescent Suicide- A Rising Trend
}

\author{
Rabindran ${ }^{1}$, Gedam DS ${ }^{2}$ \\ ${ }^{1}$ Dr. Rabindran, Consultant, Neonatologist, Billroth Hospital, Chennai, ${ }^{2}$ Dr D Sharad Gedam, Professor of Pediatrics, L N \\ Medical College, Bhopal, MP, India
}

Address for Correspondence: Dr Rabindran, E mail: rabindranindia@ yahoo.co.in

Abstract

Suicide has become one of the leading cause of death in adolecesnt age group. In this abstract we are trying to find out causes of increasing trends and preventive stratigies.

Keywords: Adolescent suicide, firearms, Suicide prevention

Suicide is the $3^{\text {rd }}$ leading cause of death among adolescents after accidents \& homicide. According to WHO, a suicide occurs every 40 seconds \& an attempt is made every 3 seconds. The ratio of attempted suicides to completed suicides among adolescents is estimated to be $50: 1$ to $100: 1$, \& the incidence of unsuccessful suicide attempts is higher among females than among males [1]. However adolescent males have 6 times higher rate of completed suicide than females because they use more lethal methods such as firearms, hanging, or jumping from heights. According to CDC, One in five teenagers in the U.S. seriously considers suicide annually. In India, adolescent suicide amount to one-third of all suicides. Overall adolescent suicide has increased $300-400 \%$ over the past 10 years [2].

The major risk factor for adolescent suicide is the presence of mental illness [3]. Adolescents have strong feelings of stress, confusion, self-doubt \& financial uncertainty. Other risk factors are family history of suicidal behaviour or mood disorder, history of physical or sexual abuse, exposure to violence, easy access to firearms, conflict with close friends or family members, use of alcohol or drugs, social isolation, academic pressure, loss of a valued relationship, struggling with sexual orientation, impaired social skills \& impaired peer relationships \& bullying. Moreover adolescents have a stigma associated with asking for help. The barriers to accessing services along with cultural \& religious beliefs are other factors associated with the rising trend among adolescent suicides.

There is an increase in firearm use among adolescents recently. Firearms were the instrument of death in $88 \%$ of teen homicides \& $41 \%$ of teen suicides in 2014 [4]. While non-firearm injuries result in death in only one out of every 760 cases, almost one in four youth firearm injuries is fatal. Alcohol use has been associated with $50 \%$ of suicides [5]. Gay \& bisexual adolescents exhibit 3 times higher suicide than others. Monozygotic twins show significantly higher concordance for suicide than dizygotic twins [6]. Increasing homosexual behaviour, alcohol abuse \& increasing twin births secondary to rise in assisted reproductive techniques nowadays could be the reason behind the rise in adolescent suicides. Overdose using over-the-counter; prescription \& nonprescription medicine is also a very common method for suicide. Media coverage of a teenage suicide may lead to cluster suicides within a 1-to 2-week period afterward [7]. Copy-cat suicides \& publicized suicides also occur frequently. Some experts believe that onechild norm may be the cause behind the rise because those adolescents are not used to dealing with difficult interpersonal problems as they grew up with no siblings.

About $80 \%$ of all suicide attempts \& completions are preceded by warning signs [2] like change in eating \& sleeping habits; withdrawal from friends, family \& regular activities; violent actions, rebellious behavior, running away; drug \& alcohol use, neglect of personal appearance, marked personality change, persistent boredom, difficulty concentrating, frequent complaints about physical symptoms, loss of interest in pleasurable activities, not tolerating praise or rewards, talking about dying, threats of suicide- either direct or indirect, overwhelming sense of guilt, shame or rejection, putting affairs in order, sudden cheerfulness after a 
period of depression, irritability, hallucinations or bizarre thoughts. It is important to identify these warning signs promptly and prevent their attempts.

Suicide prevention efforts should focus on school education programs, crisis centre hotlines, screening programs that seek to identify at-risk adolescents, media guidelines \& efforts to limit firearm access. It is important for parents and family members of adolescents with suicidal tendencies to seek professional help immediately share their feelings, encourage the teen not to isolate himself or herself from family and friends\& recommend relaxing exercises. They should address depression or anxiety seriously, pay attention \&never shrug off threats of suicide as teen melodrama \& most important listen to their words with compassion \& concern. Firearms, alcohol \& medications should be stored properly. There are many rehabilitation programs like Teen Screen Program, Stop a Suicide Today, National Suicide Prevention Lifeline which can guide these adolescents for a better future. Finally completed suicide is only the tip of the iceberg of the psychosocial crisis faced by adolescents\& hence this vulnerable period must be handled with utmost care to prevent the rising trend of adolescent suicides.

Kiran et al in this issue discussed about adolescents sucide trends. They found that Suicide among adolescents is a serious and major issue. The increasing evidence of suicidal menace is creating a greater challenge for psychiatrists, social workers, public health personnel, sociologists and psychologists to identify the underlying factors [8].
1. Husain, S.A. Current perspective on the role of psychological factors in adolescent suicide.Psychiatr Ann. 1990;20:122-127.DOI: 10.3928/0048-571319900301-06

2. Ernest J. Bordini. Child and Adolescent Suicide, Clinical Psychology Associates of North Central Florida; CPANCF.COM. 352 336-2888.

3. Friedman RA. Uncovering an epidemic-screening for mental illness in teens. N Engl J Med. 2006 Dec 28;355(26):2717-9.

4. Fingerhut, D. and Christoffel, K. (2002) Firearmrelated death and Injury among children and adolescents. The Future of Children, 12(2), 25-38.

5. Frances RJ, Franklin J, Flavin DK. Suicide and alcoholism. Am J Drug Alcohol Abuse. 1987;13:327341 .

6. Roy A, Segal NL, Centerwall BS, Robinette CD (1991) Suicide in twins. Arch Gen Psychiatry 48:29-32.

7. Gould MS, Wallenstein S, Kleinman M (1990) Timespace clustering of teenage suicides. Am J Epidemiol. 1990 Jan;131(1):71-8.

8. Kumar KK, Sattar FA, Veluswamy P, Bondade S. A Descriptive Analysis of Psychosocial Factors Associated With Nonfatal Adolescent Suicide Attempts. Int J Med Res Rev 2016;4(2):205-215. doi: 10.17511/ijmrr.2016.i02.014.

\section{Reference}

\section{How to cite this article?}

Rabindran, Gedam DS. Adolescent Suicide- A Rising Trend. Int J Med Res Rev 2016;4(2):142-143. doi: 10.17511/ijmrr.2016.i02.024. 